\title{
THE OPENING SNAP OF MITRAL STENOSIS BY
}

\author{
PATRICK MOUNSEY
}

From the Cardiac Department of the London Hospital

Received November 28, 1952

Sixteen years after Laennec (1819) described the murmur of mitral stenosis, Bouillaud (1835) described a reduplication of the second sound in mitral stenosis. The familiar cadence in mitral stenosis, which results from the presence of this added sound, was likened by Durozier (1862) to the syllables ffou-ta-ta-rou. In 1872 Guttman suggested that this sound might be due not to asynchronism of pulmonary and aortic valve closure, as had been held by Durozier, but to vibration at the stenosed mitral valve itself. Sansom (1881) held the same views as Guttman. It was not until 1888 that Rouchès introduced the term commonly used today "claquement d'ouverture de la mitrale" or opening snap of the mitral valve, to describe this sound, stating that Potain had used this term in his teaching. He suggested that the sound was produced by sudden stretching of the stenosed mitral valve by blood passing from the left auricle to the left ventricle. Rouchès' views on the pathogenesis of the opening snap were not universally accepted and Gallavardin (1905) and Duchosal (1946) postulated other mechanisms. Margolies and Wolferth (1932) made a detailed clinical and phonocardiographic study of this sound and concluded that Rouchès' hypothesis as to its mode of production was the most likely.

The clinical recognition of the opening snap and its differentiation from two other sounds in early diastole, may sometimes present difficulty. The second component of the second sound when widely split may closely resemble an opening snap. Further, the physiological third heart sound might be confused with the opening snap. The heart sounds were examined clinically and in the phonocardiogram in 33 cases of mitral stenosis to test certain clinical signs that might make easier the differentiation and recognition of these sounds.

\section{The InVestigation DesCribed}

Mitral stenosis was considered to be the principal lesion in all cases and the sole valvular lesion in 28 of them, while in four there was slight aortic incompetence and in another associated mitral incompetence. The ages of the patients varied between 19 and 48, with an average of 34 years. There were 7 men and 26 women. None had gross cardiac enlargement. The electrocardiogram showed evidence of right ventricular preponderance in 24 , and auricular fibrillation was present in 12 patients. Fifteen have since had a mitral valvotomy.

Phonocardiograms were recorded on a four-channel phonocardiograph using crystal microphones, as described by Leatham (1949). An electrocardiogram was used as a reference tracing.

\section{INCIDENCE AND RELATIONSHIP OF THE OPENING SNAP}

An opening snap was diagnosed clinically and confirmed on the phonocardiogram in 28 out of the 33 patients. In four of the remaining five the phonocardiogram recorded an opening snap. In three of these the sound was very soft and therefore difficult to recognize with certainty clinically ; in the fourth it followed closely the second heart sound and phonocardiography was necessary to analyse correctly the sequence of sounds. In only one case was an opening snap absent on the phonocardiogram. 
The term snap well described the quality of the sound heard. On the phonocardiogram the sound was composed chiefly of high frequency vibrations of short duration $(0.005$ to $0.01 \mathrm{sec}$.).
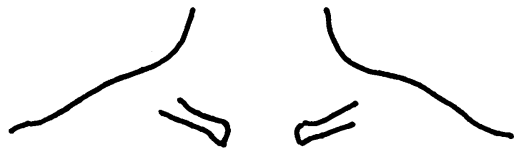

(2)

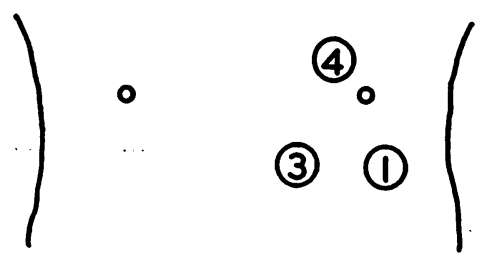

Fig. 1.-Diagram to illustrate microphone positions. (1) Mitral area. (2) Pulmonary area. (3) Left sternal edge. (4) Supramammary area.
The area where the sound was best heard was determined by examining its intensity, both on auscultation and on the phonocardiogram in four selected areas, the mitral area, the pulmonary area, the left sternal edge at the level of the fifth intercostal space, and the special supramammary area, which lies above and a little internal to the left nipple (Fig. 1). The opening snap was best heard in the supramammary area, in 27 out of 32 cases. Maximum audibility, however, was shared between two or more areas in the majority, the commonest after the supramammary area being the left sternal edge and the mitral area, and the least common the pulmonary area.

The interval between the beginning of the second sound and the opening snap varied widely. The minimum interval was $0.03 \mathrm{sec}$., the maximum $0.14 \mathrm{sec}$., and the average for the group $0.07 \mathrm{sec}$. (Fig. 2).

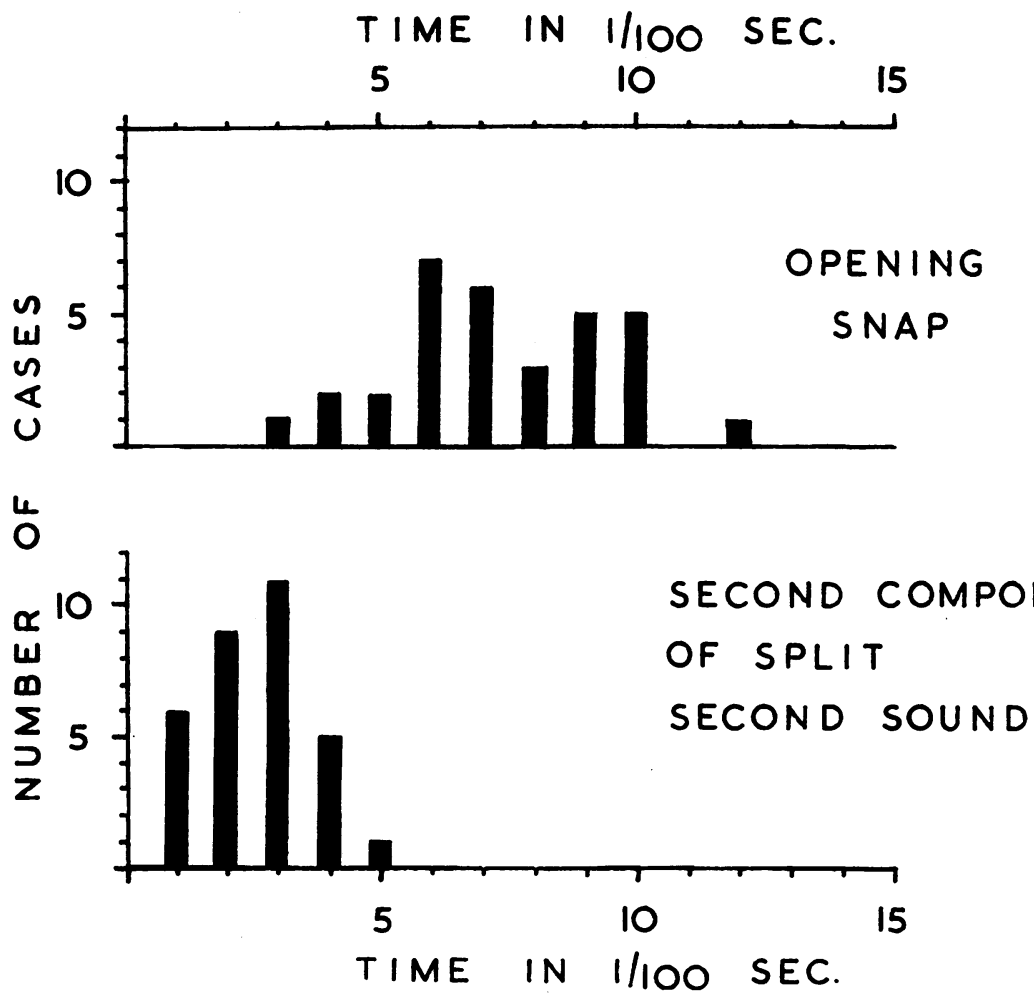

FIG. 2.-The timing of the opening snap and the second component of a split second sound compared in 33 cases of mitral stenosis. The interval between the beginning of the second sound and the opening snap varied between 0.03 and $0.12 \mathrm{sec}$. (average $0.07 \mathrm{sec}$.): that between the beginning or first component of the second sound and the second component varied between 0.01 and $0.05 \mathrm{sec}$. (average $0.03 \mathrm{sec}$.). Minimum intervals for the opening snap and maximum intervals for the second component of a split second sound lay in the same range $(0.03$ to $0.05 \mathrm{sec}$.). (The maximum interval between the beginning of the second sound and the opening snap (0.14 sec.) mentioned in the text, is not recorded in the Table, where only mean intervals for individual patients are shown.) 
In the phonocardiogram a relationship was noted between the diastolic interval on the one hand and the interval between the beginning of the second sound and the opening snap on the other in individual patients in sinus rhythm. An increase in the diastolic interval with slowing of the heart rate was accompanied by a longer interval between the beginning of the second sound and the opening snap. No similar direct correlation was seen between these two variables in the group as a whole. In individual patients with auricular fibrillation, a general relationship was noted between the preceding diastolic interval and the timing of the opening snap that followed, the snap occurring earlier after a short and later after a long diastolic interval (Fig. 3). As in those in sinus rhythm this relationship, which held generally in most subjects, did not hold for the group as a whole, where no direct correlation was noted between the diastolic interval and the timing of the opening snap.

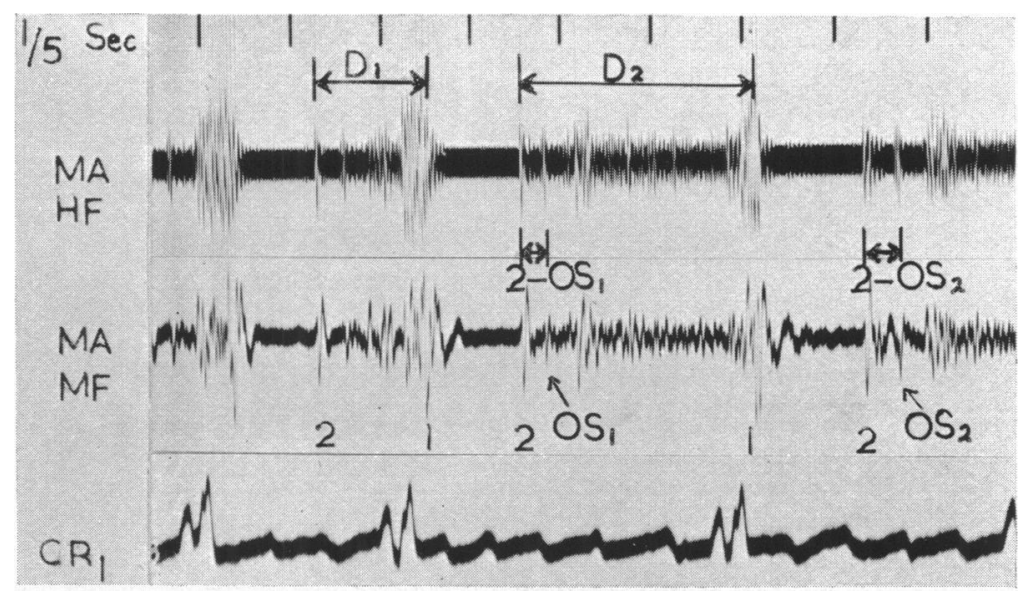

FIG. 3.-Relationship between the diastolic interval (D) and the interval between the beginning of the second sound and the opening snap (2-OS) in mitral stenosis with auricular fibrillation. A short diastolic interval $\left(D_{1}\right)$ is followed by an earlier opening snap $\left(O S_{1}\right)$ : a longer diastolic interval $\left(D_{2}\right)$ by a later opening snap $\left(\mathrm{OS}_{2}\right)$. $\mathrm{MA}=$ mitral area. $\mathrm{HF}$ and $\mathrm{MF}=$ high- and medium-frequency filters. $\quad C R=$ electrocardiographic lead $C_{1}$.

Relationship to the Mid-Diastolic Murmur. In 27 out of 32 cases, the opening snap was closely followed by the mid-diastolic murmur on the phonocardiogram (Fig. 4). In two of the remainder a silent gap was recorded between the opening snap and the murmur (Fig. 5). In three, records did not give clear information about the time of the start of the murmur. In eight cases there was an initial crescendo to the murmur, which reached its greatest intensity about $0.05 \mathrm{sec}$. after the opening snap (Fig. 4). Clinically it was often difficult to time the murmur more accurately than to say that it followed closely the opening snap.

Relationship between the Opening Snap and the Degree of Mitral Valve Disease Present. Absence or softness of the opening snap was noted in four cases in the series. In three of these gross calcification of the mitral valve was present and this was diagnosed radiologically and on palpation at valvotomy ; in the fourth the stenosis was slight, as judged by the absence of symptoms and of generalized cardiac enlargement. One further case of gross calcification of the mitral valve was met, but in this the opening snap was not unusually soft.

These findings are in agreement with the observations of Rouchès (1888) that the opening snap was not heard when the stenosis was mild or in extreme cases where the valves had lost their suppleness and ability to snap. Wynn (1952) found an association between gross calcification of the mitral valve, softness of the first heart sound, and absence of the opening snap. Sellors (1952) suggests that the snapping quality of the first heart sound in mitral stenosis may be produced at the anterior cusp of the mitral valve and notes that if a finger is placed on this cusp during the operation 


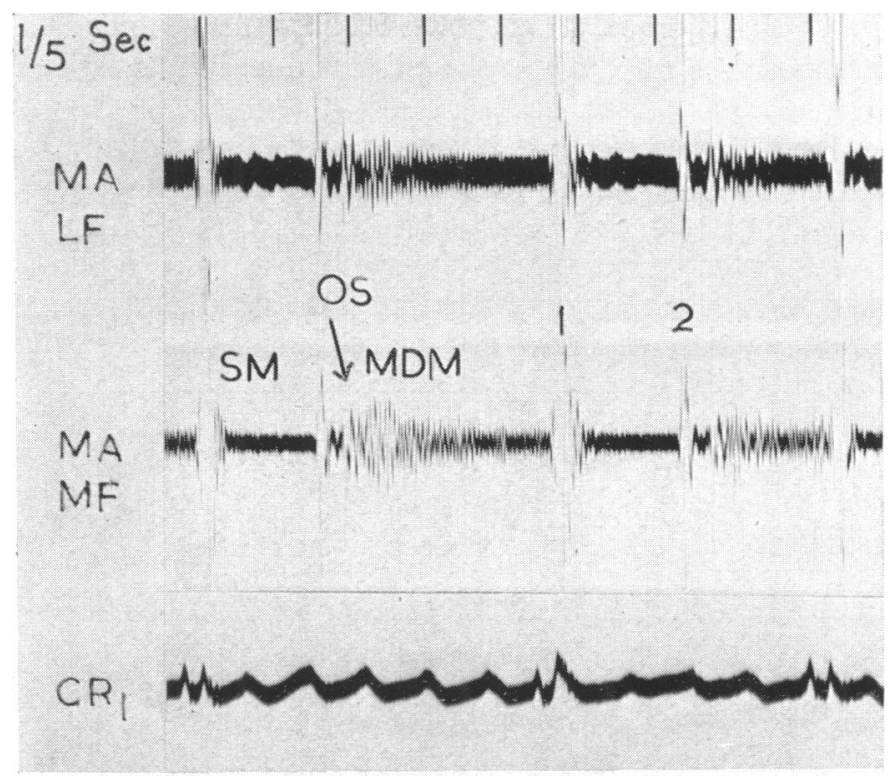

FIG. 4.-Time relationship between the opening snap (OS) and the mid-diastolic murmur (MDM). The murmur follows closely the opening snap. In the first complex there is an initial crescendo in the mid-diastolic murmur. $\mathrm{MA}=$ mitral area. $\mathrm{MF}$ and $\mathrm{LF}=$ medium- and low-frequency filters. 1 and $2=$ first and second heart sounds. $\mathbf{S M}=$ systolic murmur.

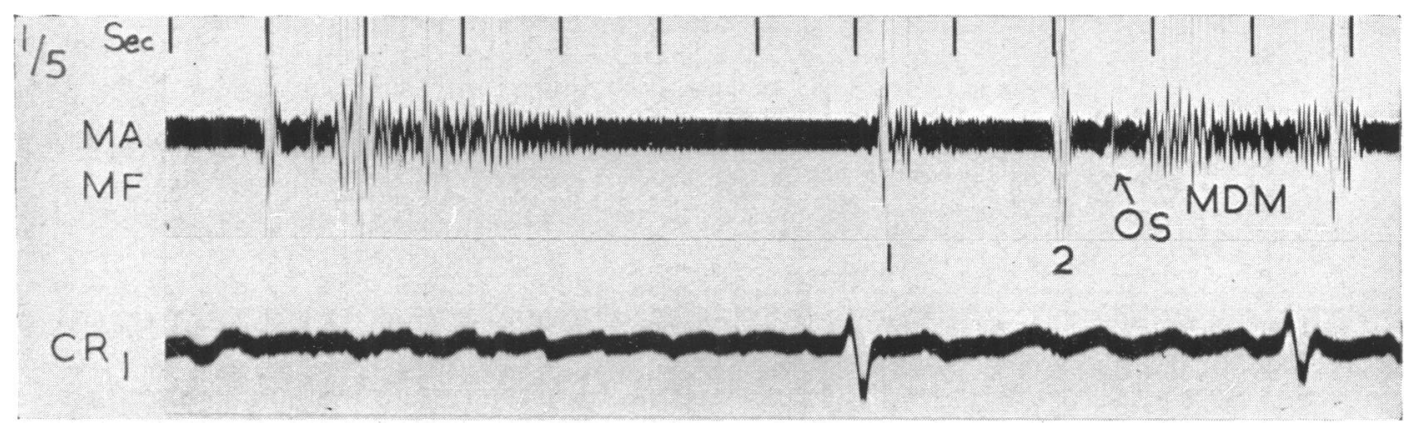

FIG. 5.-Time relationship between the opening snap and the mid-diastolic murmur. A short silent gap separates the opening snap (OS) from the beginning of the mid-diastolic murmur (MDM). MA=mitral area. $\quad M F=$ medium-frequency filter. 1 and $2=$ first and second heart sounds.

of valvotomy, as it balloons into the left atrium at the beginning of systole, the snapping quality of the sound, heard by listening directly to the heart with a stethoscope, is temporarily abolished. If, therefore, the diseased mitral valve itself gives rise both to the loud, abrupt, snapping first heart sound and to the opening snap, then the clinical association noted between the intensity of the two sounds in this series is explained.

\section{Differentiation of the Opening Snap from Splitting of the Second Sound}

Audible splitting of the second sound is physiological, provided auscultation is carried out in the pulmonary area ; the interval between the two components varies with respiration, tending to increase with inspiration (Potain, 1866 ; Leatham and Towers, 1951). 
Splitting of the second sound in the pulmonary area was recognized clinically and confirmed on a high-frequency phonocardiogram in 31 cases in this series. Phonocardiography demonstrated its presence in a further case, in which clinically it could not be differentiated from the opening snap, on account of persistent tachycardia. In the majority (31 out of 33) both splitting of the second sound and an opening snap were present in the same patient (Fig. 6). It was not felt that the quality of the second component of a split second sound enabled it always to be distinguished from an opening snap.

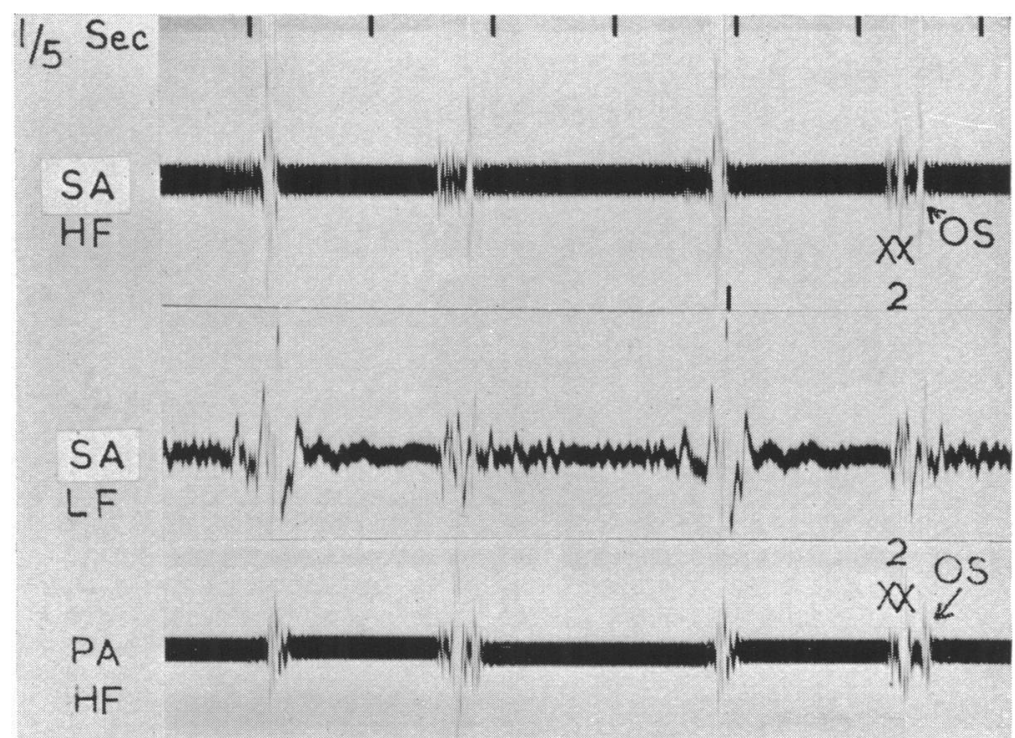

FIG. 6.-Splitting of the second sound (2xx) closely followed by the opening snap (OS). The second component of the split second sound is of greater intensity than the opening snap in the pulmonary area (PA). In the supramammary area (SA) the opening snap is the greater. HF and LF=highand low-frequency filters.

The area where the second component of the second sound was best heard differed markedly from that of the opening snap. In every case the former was loudest in the pulmonary area and in only a minority was it equally well heard in other areas.

The interval between the beginning or first component and the second component of a split second sound varied between $0.01 \mathrm{sec}$. and $0.05 \mathrm{sec}$., and the average interval for the group was $0.03 \mathrm{sec}$. This average was in contrast to the average interval between the beginning of the second sound and the opening snap $(0.07 \mathrm{sec}$.) (Fig. 2). It will be noted, however, that the maximum interval between the first and second components of the second sound and the minimum interval between the beginning of the second sound and the opening snap lay in the same range $(0.03$ to $0.05 \mathrm{sec}$.).

The effect of respiration on the width of splitting of the second sound was noted in most patients, the interval tending to lengthen on inspiration and to shorten on expiration. In six patients splitting of the second sound was only present on deep inspiration. Although phasic variation in amplitude of the opening snap was seen, the snap tending to be diminished in intensity during inspiration, no variation in its timing was noted during respiration.

\section{Differentiation of the Opening SNAP from the THIRd Heart Sound}

The criteria used for diagnosis of the third heart sound have been those put forward by Orias and Braun-Menendez (1939) and Rappaport and Sprague (1942). In quality it is predominantly 
a low-frequency sound as opposed to the higher frequency of the opening snap. The mid-point of the third heart sound lies between $0 \cdot 16$ and $0 \cdot 24 \mathrm{sec}$. after the beginning of the second sound. This is greater than the value of 0.03 to $0.14 \mathrm{sec}$. for the opening snap in this series. The third heart sound is usually best heard a little internal to the mitral area (Evans, 1942).

In no patient was a clear third heart sound recognized clinically, although a small third heart sound was present in the phonocardiogram in eight (Fig. 7). The rarity of the third heart sound in mitral stenosis is in contrast with the frequency of an opening snap.

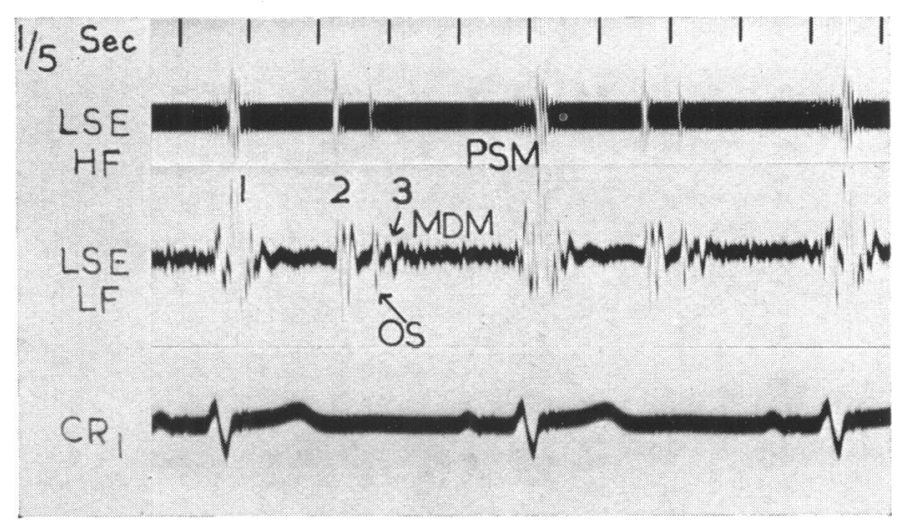

Fig. 7.-The third heart sound in the presence of an opening snap and a mid-diastolic murmur. A small third heart sound (3) is recorded during the beginning of the mid-diastolic murmur (MDM). It is preceded by the opening snap (OS). LSE=left sternal edge. $\mathrm{HF}$ and $\mathrm{LF}=$ high- and low-frequency filters. 1 and $2=$ first and second heart sounds. PSM=presystolic murmur. $\mathrm{CR} 1=$ electrocardiogram in lead CR1.

Orias and Braun-Menendez (1939) suggested that the third heart sound was due to vibrations produced during rapid ventricular filling. In mitral stenosis left ventricular filling is slow, being impeded by the stenosis, and so there is diminution or absence of a third heart sound, which is replaced by the mid-diastolic murmur. When, on the other hand, incompetence is the only lesion in mitral valve disease, Brigden and Leatham (1952) have shown that an obvious third heart sound is a frequent finding : this is explained by rapid filling of the left ventricle through the widely patent mitral valve.

\section{Discussion}

In this series clinical differentiation between an opening snap and the second component of a split second sound was generally possible, based on the site where the sound was best heard and on the interval that separated it from the first component of the second sound. The fact that in most cases both sounds were present was of great value in distinguishing each. Deep breathing was a manœuvre that sometimes aided clinical distinction between the sounds, since with this the splitting of the second sound tended to widen, or in some cases to appear de novo, while the intensity of the opening snap tended to diminish.

Cases with persistent tachycardia presented the greatest difficulty, for in these splitting of the second sound and an opening snap lay crowded into an interval of $0.03 \mathrm{sec}$. When carotid pressure induced a slower heart rate, differentiation of these three sounds was facilitated. In one patient, in whom this manœuvre failed, phonocardiography with a rapidly moving film showed the presence of both splitting of the second sound and an opening snap.

The differential diagnosis of the opening snap from a third heart sound seldom caused difficulty. Although a small third heart sound was recorded phonocardiographically in eight cases, clinically it was never audible. 
The opening snap was found to be most useful for the diagnosis of mitral stenosis in patients in whom other signs were missing on casual examination. When the opening snap was heard by itself, it stimulated a more thorough search for a latent mid-diastolic murmur.

Although the pathogenesis of the opening snap remains in doubt, there is evidence that it is produced at the stenosed mitral valve itself. Margolies and Wolferth (1932) showed that the opening snap coincided with the beginning of the descent of the $\mathrm{V}$ wave in a jugular pulse tracing. Assuming that the tricuspid and mitral valves open almost simultaneously, the fall in jugular venous pressure following the opening snap strongly suggests that this sound is coincident with the opening of the mitral valve.

The association of the opening snap with mitral stenosis alone and its absence in other diseases causing pulmonary hypertension or pulmonary venous congestion is an argument in favour of its production at the stenosed mitral valve. Its tendency to become softer or disappear with extreme rigid calcified stenosis suggested that it is not directly related to any increase in left auricular pressure, which would remain unrelieved or tend to increase further with increasing stenosis, but rather to the state of the mitral valve itself. Its infrequency in early mitral valve disease when the diastolic murmur first appears (Thomas, 1952) suggests that considerable stenosis is required to produce it. Its absence in pure mitral incompetence (Guttman, 1872 ; Brigden and Leatham, 1952) emphasizes its essential connection with a stenotic lesion.

If it be assumed that the opening snap is produced at the mitral valve, then hæmodynamic factors may be used to explain its variable timing. The timing of the opening snap depends on the inter-relationship between the left atrial pressure and the rate of fall of left ventricular pressure during the phase of isometric relaxation. When the left ventricular pressure falls below the left atrial, the mitral valve opens with the production of the opening snap. Messer et al. (1951) have explained in the following way the finding that in patients in auricular fibrillation a short cardiac cycle is followed by an early opening snap. A short diastolic interval in mitral stenosis tends to cause an increase in left atrial pressure, due to inefficient emptying of the left atrium. In the subsequent diastole, therefore, left atrial pressure exceeds left ventricular pressure slightly earlier and hence the mitral valve opens earlier. The occurrence of an earlier opening snap, with increasing heart rate and hence shorter diastolic interval, in patients in sinus rhythm could be similarly explained. The diastolic interval, however, is only one of many factors affecting the left atrial pressure and left atrial pressure is not the only factor affecting the timing of the opening snap. Although, therefore, a relationship was seen in individual cases in our series between the diastolic interval and the timing of the opening snap, in the group as a whole no direct relationship was found.

\section{SUMMARY}

A clinical and phonocardiographic study of 33 cases of mitral stenosis has been made. An opening snap was heard in 28: in a further 4 it was recorded on the phonocardiogram although not detected clinically; in these its soft character or the presence of tachycardia caused it to be missed clinically. In only one case was an opening snap absent in the phonocardiogram.

Differentiation of the opening snap from splitting of the second sound was made on the timing of the sounds and the area where they were best heard. The second component of a split second sound was always loudest in the pulmonary area and the opening snap usually loudest in the area above and internal to the left breast. The frequency with which both sounds were present (31 out of 33) made their recognition relatively easy. Phonocardiography was necessary to differentiate between them in only one case.

The physiological third heart sound differs from the opening snap in its low-pitched quality, the area where it is best heard and its timing. In no patient was a clear third heart sound recognized clinically, although in eight a small third heart sound was present in the phonocardiogram.

Absence or diminution in intensity of the opening snap was associated with gross calcification of the mitral valve in three cases and with slight stenosis in a fourth. 
The opening snap was found to be a useful diagnostic sign, when other signs of mitral stenosis were missing on casual examination. Its presence should lead to a more thorough search, which should reveal the latent mid-diastolic murmur.

I would like to acknowledge with sincere thanks the help and criticism of Dr. William Evans in the preparation of this paper. I wish to thank Mr. W. D. Dicks for technical help with the phonocardiograms.

\section{REFERENCES}

Bouillaud, J. (1835). Traité clinique des Maladies du Crour, précédé de Recherches nouvelles sur l'Anatomie et la Physiologie de cet Organe. Baillière, Paris.

Brigden, W. W., and Leatham, A. (1953). Brit. Heart J., 15, 55.

Duchosal, P. (1946). Praxis, 24, 385.

Durozier, P. (1862). Arch. gén. Méd. 20, 385.

Evans, W. (1942). Brit. Heart J., 5, 205.

Gallavardin, L. (1905). Lyon méd., 105, 401.

Guttman, P. (1872). Lehrbuch der Klinischen Untersuchung Methoden. Hirschwald, Berlin, 257.

Laennec, R. T. H. (1819). De l'Auscultation médiate ou Traité du Diagnostic des Maladies des Poumons et du Coeur, fondé principalement sur ce nouveau Moyen d'Exploration. Brosson et Chaudé, Paris.

Leatham, A. (1949). Post. Grad. med. J., 25, 568.

, and Towers, M. (1951). Brit. Heart J., 13, 575.

Margolies, A., and Wolferth, C. C. (1932). Amer. Heart J., 7, 443.

Messer, A. L., Counihan, T. B., Rappaport, M. B., and Sprague, H. B. (1951). Circulation, 4, 576.

Orias, O., and Braun-Menendez, E. (1939). The Heart Sounds in Normal and Pathological Conditions. New York. Potain, C. (1866). Bull. Soc. méd. Hôp. Paris, 3, 138.

Rappaport, M. B., and Sprague, H. B. (1942). Amer. Heart J., 23, 591.

Rouchès, F. J. M. (1888). Thèse de Paris.

Sansom, A. E. (1881). Proc. Med. Soc. Lon., 5, 191.

Sellors, T. H. (1952). Personal communication.

Thomas, G. T. (1952). Personal communication.

Wynn, A. (1952). Brit. Heart J., 14, 296. 